\title{
The Effect of the Chemical Potentials of Electrodes on Charge Transport across Molecular Junctions
}

Geng-Min Lin, ${ }^{\dagger} \S$ Chih-Hsun Lin, ${ }^{\dagger,}$ Hao Howard Peng, ${ }^{\dagger} \S$ Han Hsiao, ${ }^{\dagger}$ Tsai-Hui Wang, ${ }^{\ddagger}$ Ching-Hwa Ho, ${ }^{, *}$ Hsiu-Fu Hsu, ${ }^{\neq}, *$ and Chun-hsien Chen ${ }^{\dagger, *}$

†Department of Chemistry and Center for Emerging Material and Advanced Device, National Taiwan University, Taipei, Taiwan 10617.

‡Department of Chemistry, Tamkang University, New Taipei City, Taiwan 25137.

§Contributed equally to this work. *e-mail: r03223107@ntu.edu.tw; hhsu@mail.tku.edu.tw; chhchen@ntu.edu.tw

1. Experimental. S2

1-1. Electrochemical scanning tunneling microscope break junction. ........................................ S2

1-2. Homemade organic phase $\mathrm{Ag} / \mathrm{AgCl}$ reference electrode and Luggin capillary. ..................... S3

Figure S1. Homemade organic phase reference electrode and Luggin capillary. .........................S3

Table S1. Stability of homemade organic phase $\mathrm{Ag} / \mathrm{AgCl}(s)$ reference electrode. ...................... S4

2. Histograms of single-molecule conductance at $V_{w k}$ of $-0.5 \mathrm{~V}$ and $0.5 \mathrm{~V}$ by EC-STM bj. ....... S5

Figure S2. Conductance histograms of alkanediamines at $V_{\mathrm{wk}}$ of $-0.5 \mathrm{~V}$ and $0.5 \mathrm{~V}$................ S5

Figure S3. Conductance histograms of alkanedithiols at $V_{\mathrm{wk}}$ of $-0.5 \mathrm{~V}$ and $0.5 \mathrm{~V}$................... S6

Figure S4. Conductance histograms of OPEn at $V_{\mathrm{wk}}$ of $-0.5 \mathrm{~V}$ and $0.5 \mathrm{~V}$. ............................. S6

Figure S5. Conductance histograms of OPE1 at $V_{\mathrm{wk}}$ of $-0.5 \mathrm{~V}$ and $0.5 \mathrm{~V}$............................... S7

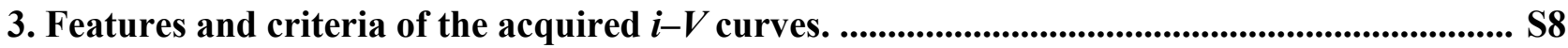

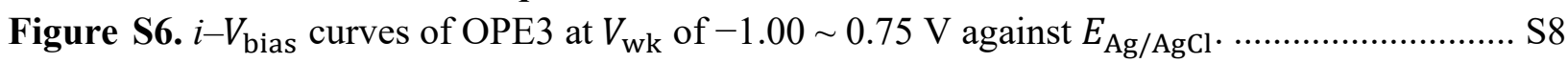

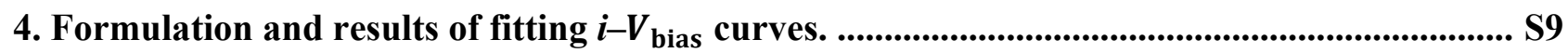

Table S2. Results of fitting $i-V_{\text {bias }}$ curves of OPE3 to the single-level Breit-Wigner formula. ... S10

Figure S7. $V_{\mathrm{wk}}$-dependent $E_{\mathrm{FMO}}^{\mathrm{o}}\left(\mathrm{V} v s . E_{\mathrm{Ag} / \mathrm{AgCl}}\right)$ from the SLBW model. ................................ S10

Table S3. Energy offset $\left(E_{\mathrm{FMO} @ V_{\text {bias }}}-E_{\mathrm{F}, \text { substrate }}\right)$ derived via $E_{\mathrm{FMO}}^{\mathrm{o}}+\gamma e V_{\mathrm{bias}}-E_{\mathrm{F}} \ldots \ldots \ldots \ldots \ldots . \mathrm{S} 11$

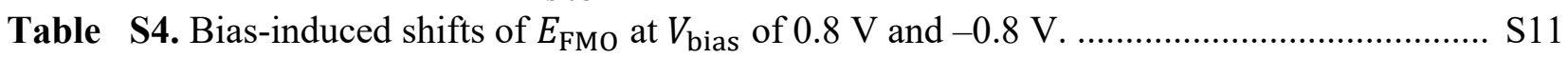

Figure S8. Transmission spectra for $i-V_{\mathrm{bias}}$ reproduction of OPE3 ...................................... S12

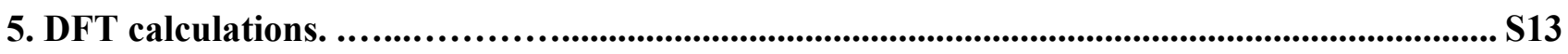

Figure S9. Optimized structures and isosurfaces of single-molecule junctions. ......................... S13

Figure S10. $V_{\text {bias }}$-dependent transmission spectra of OPE3 .................................................. S13

6. Derivation of dependency of $\boldsymbol{G}_{\text {contact }}$ and $\boldsymbol{\beta}$ on the chemical potential of the electrode. ........ S14

Figure S11. Schematic diagram of one-dimensional potential barrier. ...................................... S14

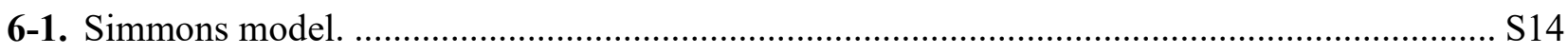

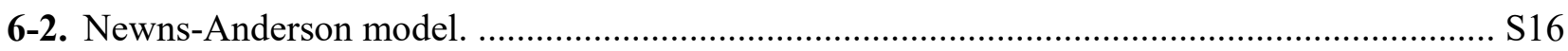

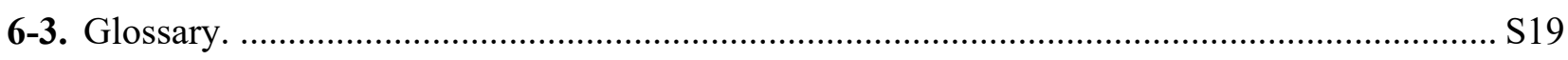

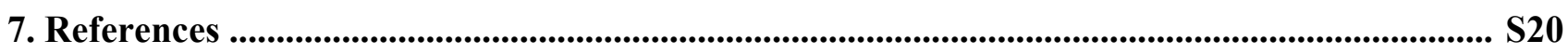




\section{Experimental}

\section{1-1. Electrochemical scanning tunneling microscope break junction.}

Experiments of electrochemical scanning tunneling microscope (EC-STM) were carried out via NanoScopeIIID controller equipped with a bipotentiostat (Universal Bipotentiostat, Veeco Instruments). The current passing across the junctions, the electrochemical potential $\left(V_{\mathrm{wk}}\right)$, and the bias voltage $\left(V_{\mathrm{bias}}\right)$ were obtained by a data acquisition system with a 24-bit PCI-4461 card driven by customized LabVIEW programs (National Instruments) at a rate of 10,000 Hz for EC-STM bj (break junction) experiments and at a rate of $40,000 \mathrm{~Hz}$ for $i-V_{\mathrm{wk}}$ and $i-V_{\mathrm{bias}}$ scans.

To perform $i-V_{\mathrm{wk}}$ and $i-V_{\mathrm{bias}}$ scans, the corresponding junction conductance was pre-determined by EC-STM bj with a rate of $50 \mathrm{~nm} / \mathrm{s}$ for the tip movement. To form single-molecule junctions, the feedback loop was deactivated and the current as well as the conductance were monitored as a function of tipmovement distance, $i(\mathrm{~s})$ and $G(\mathrm{~s})$. The tip was approached toward the substrate until the conductance was larger than $1 \mathrm{G}_{0}$ and subsequently the tip was retracted from the substrate. When the monitored $G(\mathrm{~s})$ reached the range of the pre-determined single-molecule conductance, indicating the formation of molecule junctions, the movement of the tip was stopped and immediately two cycles of the $V_{\mathrm{wk}}{ }^{-}$ waveforms shown in Scheme $1 \mathrm{~b}$ and Scheme 2a of the main text were applied, respectively, for the $i-V_{\mathrm{wk}}$ and $i-V_{\text {bias }}$ scans. The tip was then retracted to break the junction and the monitored conductance dropped to the noise level $\left(<10^{-6} \mathrm{G}_{0}\right)$. The sweeping rate was $100 \mathrm{~V} / \mathrm{s}$. For $i-V_{\mathrm{wk}}$ scans, potentials of both STM tip $\left(V_{\text {STM tip }}\right)$ and substrate $\left(V_{\text {substrate }}\right)$ were simultaneously modulated by a bipotentiostat with a constant bias $\left(V_{\text {bias }}=V_{\text {STM tip }}-V_{\text {substrate }}=50 \mathrm{mV}\right)$. For an $i-V_{\text {bias }}$ scan, $V_{\text {wk }}\left(v i z\right.$., $\left.V_{\text {substrate }}\right)$ was fixed and the bias $\left(V_{\text {bias }}=V_{\text {STM tip }}-V_{\text {substrate }}\right)$ was applied via a PCI-4461 card (vide supra).

To conduct EC-STM experiments, it is necessary to suppress the unwanted background current. Hence, insulation of the STM tip by EVA (ethylene vinyl acetate) was employed to reduce the exposed area and to minimize the leakage current. EVA, a thermoplastics material, was placed and heated on a U-shaped notch (1-mm in width) of a copper plate. When EVA melted, an STM tip with the apex facing up was positioned into the EVA-filled U-shaped notch and then was removed quickly and vertically downwards. To maintain the solute concentrations throughout experiments of typically $3 \sim 4 \mathrm{hrs,} \mathrm{PC}$ (propylene carbonate) was utilized because of its low-vapor pressure and high dielectrics to dissolve electrolytes. The appearances of the EVA-coated apices and the reasonable resistance of EVA to the dissolving power of PC before and after experiments can be found in our previous publication. ${ }^{1}$

To have a good control of the electrochemical potential for the working electrode, the area of the working electrode relative to that of the counter electrode should be small. Therefore, an EVA sheet (1.0 
$\mathrm{mm}$ in thickness) in which a tiny hole was punched and would be placed underneath the STM tip was heated on the gold substrate to reduce the active area of the working electrode.

\section{1-2. Homemade organic phase $\mathrm{Ag} / \mathrm{AgCl}$ reference electrode and Luggin capillary.}

To obtain an AgCl-electroplated silver wire for a reference electrode, a silver wire $(0.25-\mathrm{mm}$ in diameter) was cleaned by soaking in $3 \mathrm{M} \mathrm{HNO}_{3}(a q)$ for 3 min to remove native oxides and followed by rising with copious Millipore-Q purified water $(18.2 \mathrm{M} \Omega \cdot \mathrm{cm})$. With a $3.0-\mathrm{V}$ battery, $\mathrm{AgCl}(s)$ was anodically plated in $1.0 \mathrm{M} \mathrm{HCl}(a q)$. The $\mathrm{Ag} / \mathrm{AgCl}$ wire was placed into a capillary tubing (inner diameter $\sim 1.5-\mathrm{mm}$ ) in which a pencil lead (grade 4B, Pilot) was inserted slightly into the open side (see Figure S1). The open side was sealed by flame-annealing and the extruding pencil lead outside the tubing was cut. A PC solution containing the supporting electrolyte such as $0.1 \mathrm{M} \mathrm{TBAC}$ (tetrabutylammonium chloride) was filled into the capillary tubing with the pencil lead as the frit. Hence, an organic phase $\mathrm{Ag} / \mathrm{AgCl}$ reference electrode was obtained (Figure S1a).

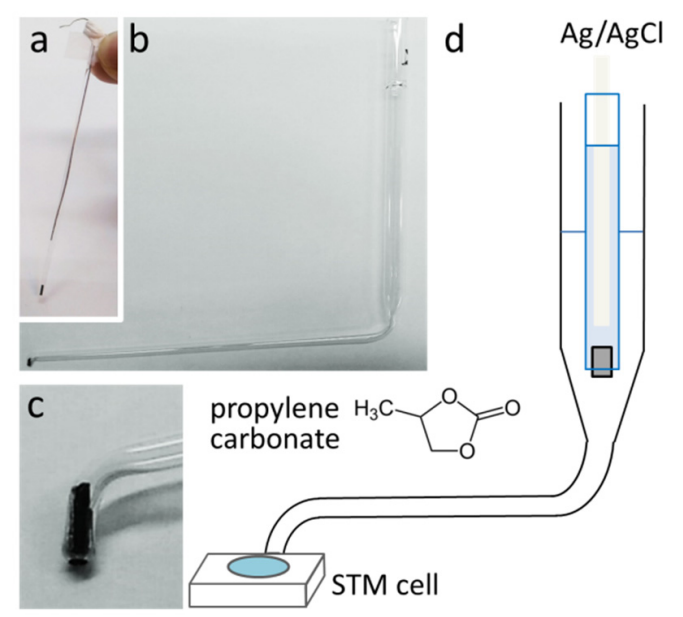

Figure S1. Homemade organic phase reference electrode and Luggin capillary. A small Lugging capillary was prepared to fit the small spacing of the EC-STM cell. Photographs of (a) a homemade reference electrode, (b) a Luggin capillary, (c) a close-up view of the end of a Luggin capillary with a pencil lead as the frit. (d) A scheme diagram illustrating the experimental setup. In this study, the solvent was propylene carbonate. The supporting electrolyte was chloride-containing $\left(\left(n-\mathrm{C}_{4} \mathrm{H}_{9}\right)_{4} \mathrm{~N}\right) \mathrm{Cl}$ in the reference electrode and was $\left(\left(n-\mathrm{C}_{4} \mathrm{H}_{9}\right)_{4} \mathrm{~N}\right)\left(\mathrm{ClO}_{4}\right)$ in both the STM cell and the Luggin capillary.

To fit the stringent geometry of the STM cell and to have the sensing point of the reference electrode close to the working electrode, employed herein was a Luggin capillary (Figure S1b and c) with a $90^{\circ}$ turn at the end. The solution for the Luggin capillary was the blank solution in the STM cell, specifically, PC with a non-halide supporting electrolyte $\left(\right.$ e.g., $\left.\left(\left(n-\mathrm{C}_{4} \mathrm{H}_{9}\right){ }_{4} \mathrm{~N}\right)\left(\mathrm{ClO}_{4}\right)\right)$. Therefore, the use of a Luggin capillary could reduce the possibility of strong halide adsorption or even etching on the gold tip and substrate due to the leakage of chloride from the reference electrode. 
To examine the potential stability of the homemade reference electrode, cyclic voltammetry was carried out with the presence of decamethylferrocene (DMFc). Table 1 summarizes $E_{\frac{1}{2}}$ of DMFc in PC. The results show that the magnitude of $E_{\frac{1}{2}}$ drifting was quite small, within $6 \mathrm{mV}$ in 5 consecutive hours. The day-to-day variation was as small as $6 \mathrm{mV}$, suggesting a stable and reliable reference electrode for our experiments.

Table S1. Stability of homemade organic phase $\mathrm{Ag} / \mathrm{AgCl}(s)$ reference electrode.

\begin{tabular}{c|rrrrrr}
\hline \multicolumn{1}{c}{} & \multicolumn{7}{c}{$E_{1 / 2}$ of DMFc $\left(\mathrm{mV} v s . E_{\mathrm{Ag} / \mathrm{AgCl}}\right)$} \\
\hline duration & day 1 & day 2 & day 3 & day 4 & day 5 & day 6 \\
\hline initial & 14 & 10 & 15 & 15 & 13 & 16 \\
$1 \mathrm{hr}$ & 11 & 6 & 11 & 8 & 11 & 11 \\
$3 \mathrm{hrs}$ & 11 & 7 & 11 & 10 & 10 & 11 \\
$5 \mathrm{hrs}$ & 8 & 8 & 9 & 9 & 10 & 11 \\
\hline
\end{tabular}




\section{Histograms of single-molecule conductance at $V_{w \mathrm{k}}$ of $-0.5 \mathrm{~V}$ and $0.5 \mathrm{~V}$ by EC-STM bj.}

This section displays raw data of conductance histograms for the values reported in Table 1 of the main text. The histograms were prepared without data selection. In the case of OPE1 (Figure S4a and b), the conductance peak was broad and had a shoulder. What were reported in Table 1 for OPE1 was presented in Panels e and f of Figure S5 which were plotted by using the first $0.1 \sim 0.2 \mathrm{~nm}$ segment of each $i-\mathrm{s}$ trace after the formation of junctions.
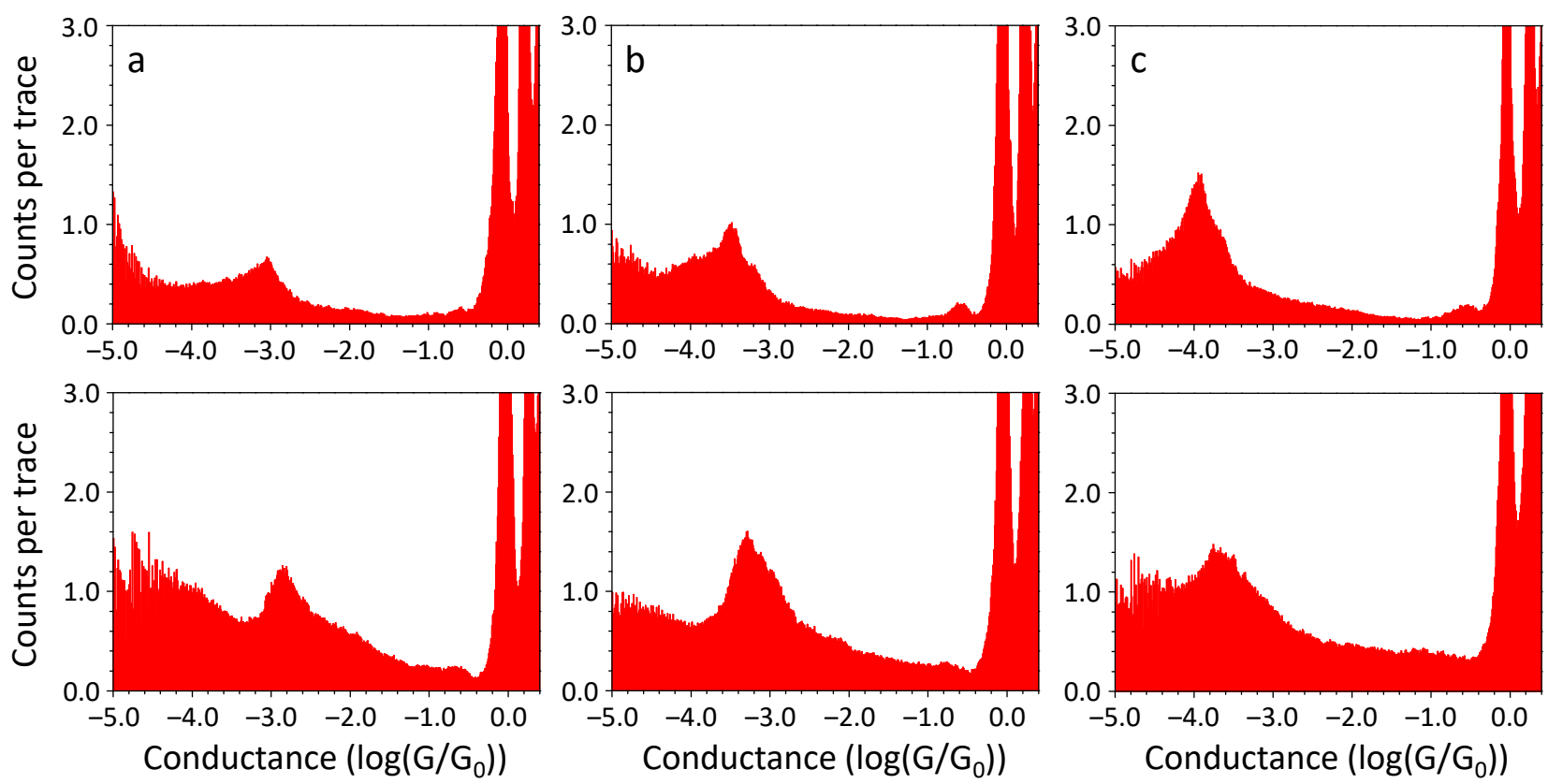

Figure S2. Conductance histograms of alkanediamines at $V_{\mathrm{wk}}$ of $-0.5 \mathrm{~V}$ (upper row) and $0.5 \mathrm{~V}$ (lower row). 1D histograms of (a) 1,4-butanediamine, (b) 1,5-pentanediamine, and (c) 1,6-hexanediamine. The molecular junctions were obtained by EC-STM bj with a gold STM tip. The working, counter, and reference electrodes were 100-nm thick gold film, a Pt wire, and an organic phase $\mathrm{Ag} / \mathrm{AgCl}(s)$ electrode, respectively. All $i-\mathrm{s}$ traces obtained were pooled to plot the histogram without data selection. The numbers of $i-\mathrm{s}$ traces to plot upper panels a, b, and c are 2002, 2102, and 2203, and for lower panels are 2029, 2027, and 2076, respectively. Conditions: $V_{\text {bias }}, 50 \mathrm{mV}$; solution, solute in propylene carbonate with the supporting electrolyte of $0.1 \mathrm{M}\left(\left(n-\mathrm{C}_{4} \mathrm{H}_{9}\right)_{4} \mathrm{~N}\right)\left(\mathrm{ClO}_{4}\right)$. 

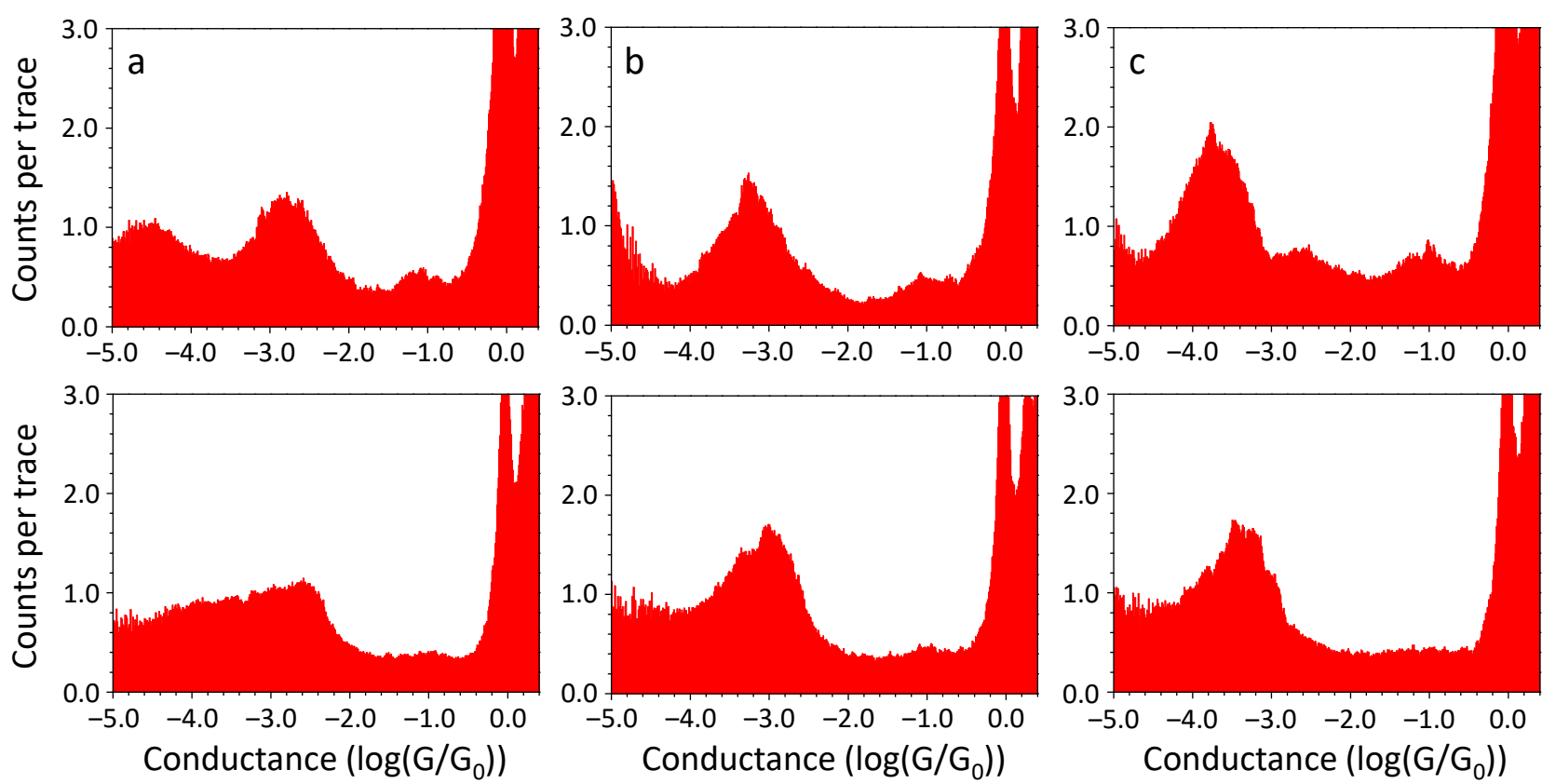

Figure S3. Conductance histograms of alkanedithiols at $V_{\mathrm{wk}}$ of $-0.5 \mathrm{~V}$ (upper row) and $0.5 \mathrm{~V}$ (lower row). 1D histograms of (a) 1,4-butanedithiol, (b) 1,5-pentanedithiol, and (c) 1,6-hexanedithiol. The numbers of $i-\mathrm{s}$ traces to plot upper panels a, b, and c are 2035, 2031, and 2037, and for lower panels are 2244, 2041, and 2036, respectively. Other conditions were the same as those of Figure S2. The conductance peak at $\sim 0.1 \mathrm{G}_{0}$ might be attributed to the formation of electrode-sulfur-electrode configurations in which the molecular terminal atom was inserted into the gold contact upon junction formation. This explanation is proposed by González and coworkers ${ }^{2}$ and they also pointed out that the measurement-to-measurement variations for the significance of this conductance peak were high (see Figure S2 of Reference 2).
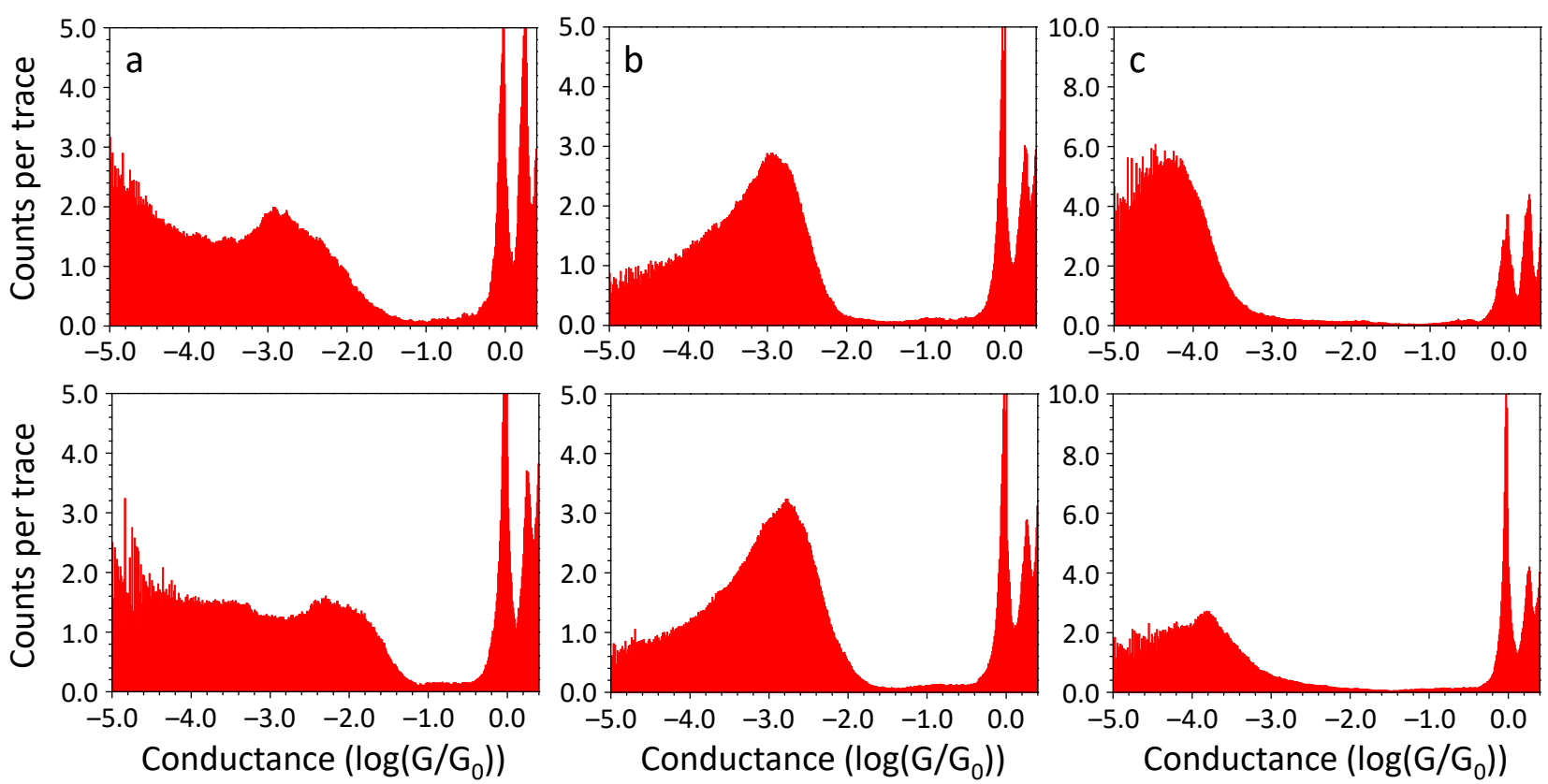

Figure S4. Conductance histograms of OPEn at $V_{\mathrm{wk}}$ of $-0.5 \mathrm{~V}$ (upper row) and $0.5 \mathrm{~V}$ (lower row). 1D histograms of (a) OPE1, (b) OPE2, and (c) OPE3. The numbers of $i$-s traces to plot upper panels a, b, and $\mathrm{c}$ are 2104, 2106, and 2169, and for lower panels are 2165, 3082, and 2461, respectively. Other conditions were the same as those of Figure S2. 

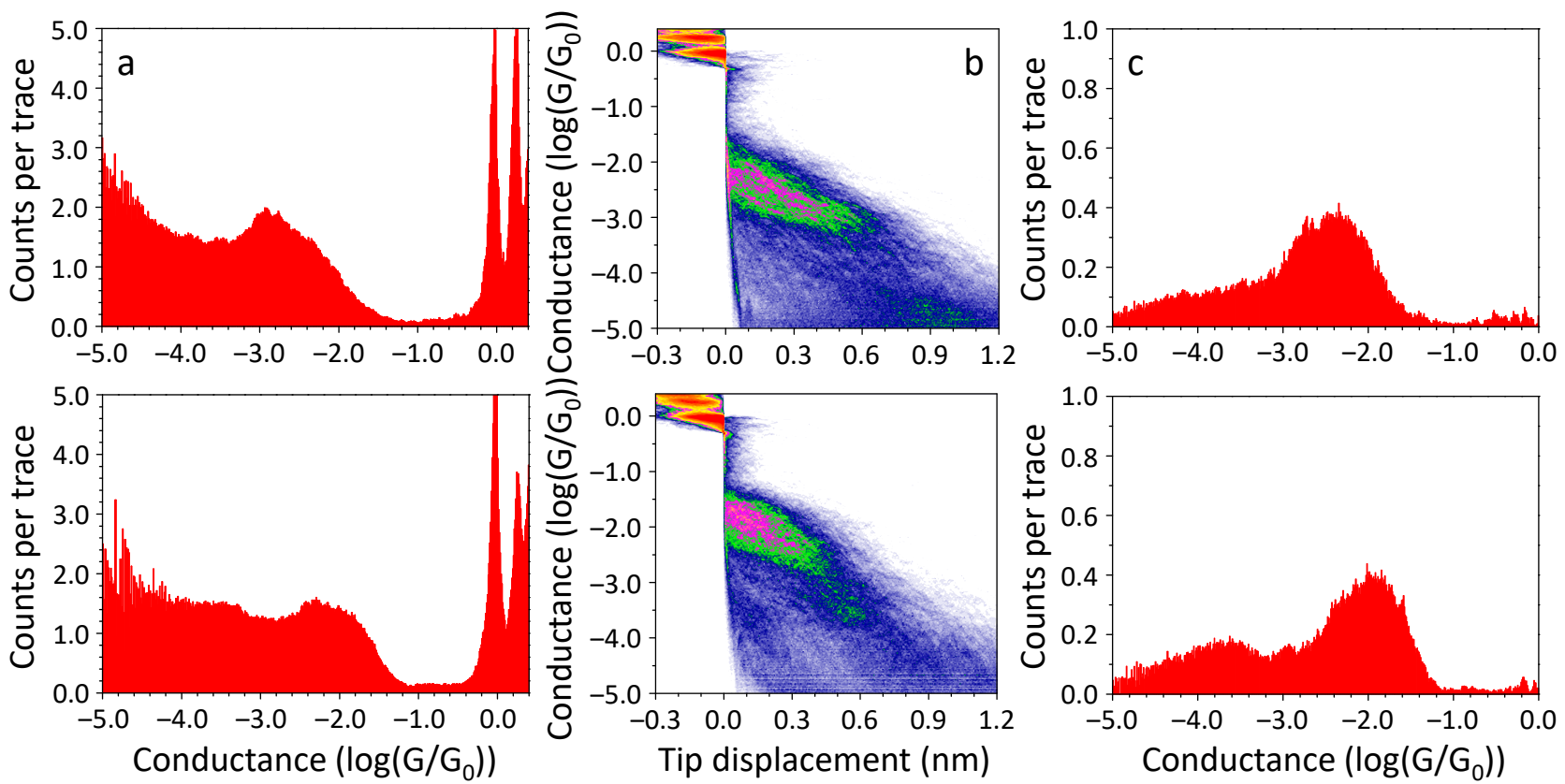

Figure S5. Conductance histograms of OPE1 at $V_{\mathrm{wk}}$ of $-0.5 \mathrm{~V}$ (upper row) and $0.5 \mathrm{~V}$ (lower row). (a) 1D histograms and (b) 2D histograms plotted with all $i-$ s traces obtained. (c) 1D histogram plotted with the $0.1 \sim 0.2-\mathrm{nm}$ portion of the tip displacement in Panel $\mathrm{b}$ due to the broadness and difficulty in the determination of the conduction values from Panels $a$ and $b$. 


\section{Features and criteria of the acquire $i-V$ curves.}

For overlaid diagrams of $i-V_{\mathrm{wk}}$ curves and $i-V_{\mathrm{bias}}$ curves without selection, it was difficult to observe the features because there were many incomplete scans due to the short lifetimes of singlemolecular junctions. Hence, with the informative two-cycle waveforms (e.g., Figure 3a), the following describes the criteria applied to decide whether the $i-V$ curve validly corresponded to a molecular junction. Firstly, the span of the conductance peak obtained by EC-STM bj was the caliber for $i-V$ curves obtained under the same conditions (such as the same $V_{\mathrm{bias}}$ or $V_{\mathrm{wk}}$ ). Curves with the junction conductance at the level of background or thru-space tunneling, viz., from junctions without containing molecules, would thus be excluded. Secondly, within the waveform, the sections that the conductance was examined were the initial holding stage (viz., at the $50-\mathrm{mV} V_{\text {bias }}$ for $100 \mathrm{msec}$ ), the consecutive 2 returns (viz., reversed scans and back to the initial conditions), and the final holding stage (for $50 \mathrm{msec}$ ) prior to the preprogrammed breakage of the junction.
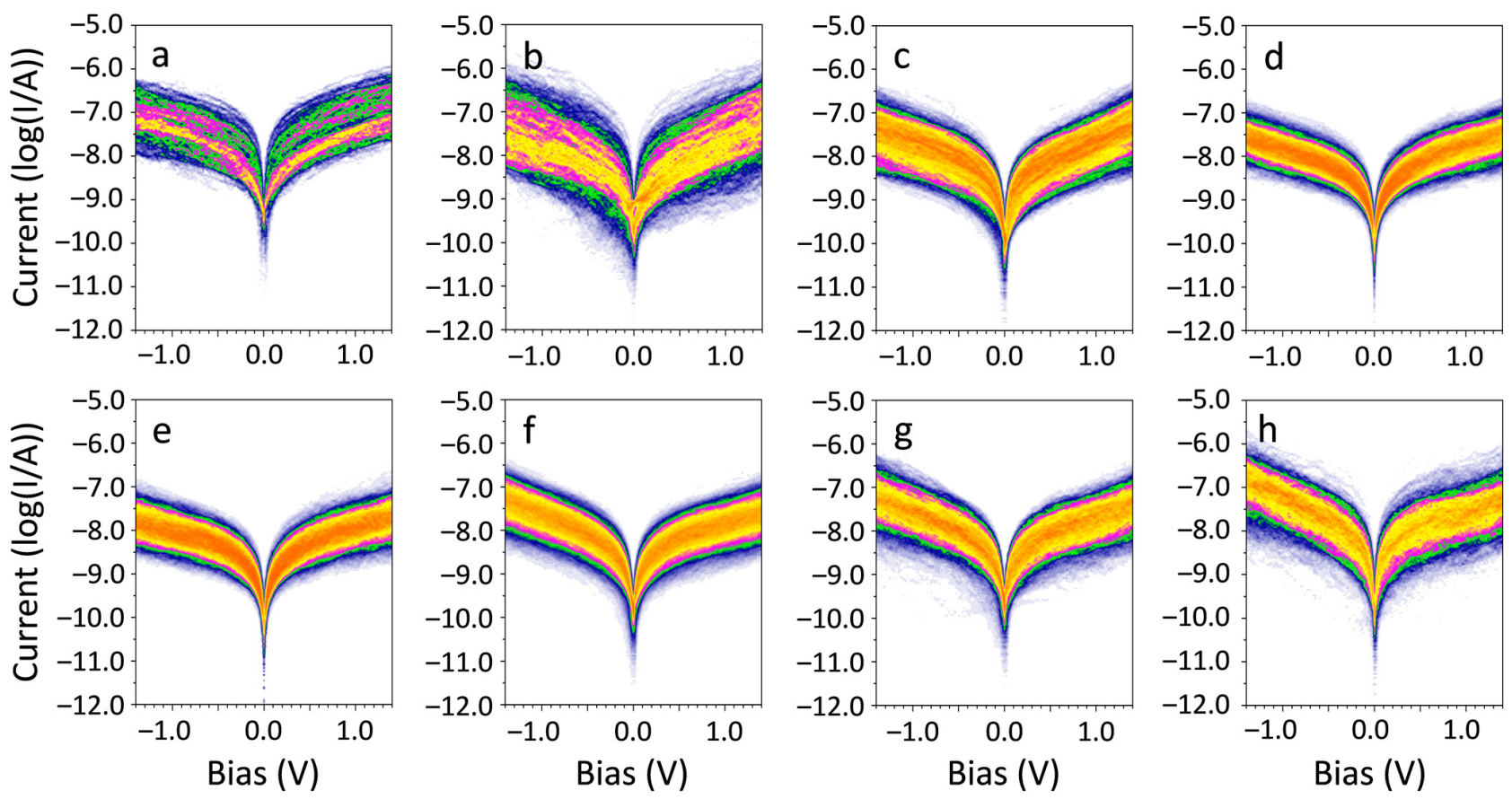

Figure S6. $i-V_{\text {bias }}$ curves of OPE3 at $V_{\mathrm{wk}}$ of $-1.00 \sim 0.75 \mathrm{~V}$ against $E_{\mathrm{Ag} / \mathrm{AgCl}}$. 2D histograms of OPE3 with $V_{\text {wk }}$ at (a) $-1.00 \mathrm{~V}$, (b) $-0.75 \mathrm{~V}$, (c) $-0.50 \mathrm{~V}$, (d) $-0.25 \mathrm{~V}$, (e) $0.00 \mathrm{~V}$, (f) $0.25 \mathrm{~V}$, (g) $0.50 \mathrm{~V}$, and (h) 0.75 $\mathrm{V}$ versus $E_{\mathrm{Ag} / \mathrm{AgCl}}$. The numbers of $i-V_{\text {bias }}$ traces to plot Panels a-h are $262,781,1674,1877,2019,4067$, 1430 , and 1027 , respectively. It was very difficult to obtain unbroken $i-V_{\text {bias }}$ curves at very positive or negative potentials. For example, the quality of curves at $V_{\mathrm{wk}}=-1.00 \mathrm{~V}$ (Panel a) was far inferior to others although extra efforts and many more runs had been carried out and, therefore, it was not utilized for the fitting to the SLBW formula (i.e., Figure S7). Other conditions were the same as those of Figure S2. 


\section{Formulation and results of fitting $i-V_{\text {bias }}$ curves.}

To obtain electronic structures under $V_{\mathrm{wk}}$ examined in this work, the $\mathrm{i}-V_{\mathrm{bias}}$ curves were fitted by the Landauer formula based on the SLBW model (see Equation 14 in the main text). Since in our system both the tip and substrate were potentiostatted with respect to $E_{\mathrm{Ag} / \mathrm{AgCl}}$, the bias window was developed in a manner illustrated in Figure 3a and different from that of the typical 2-electrode configuration. In order to calculate the current, the equation that modifies the lower and upper bounds in Landauer formula is presented below. Note that the lower bound refers to the chemical potential of the substrate $\left(E_{\mathrm{F}, \text { substrate }}\right)$ which is fixed and set to be zero; the upper bound denotes the integration range by the chemical potential of the tip $\left(E_{\mathrm{F}, \mathrm{tip}}\right)$.

$$
I=\frac{2 e}{h} \int_{0}^{e V} \frac{\Gamma_{L} \Gamma_{R}}{\left[E-\left(E_{\mathrm{FMO}}^{0}+\gamma e V\right)\right]^{2}+\left(\frac{\Gamma_{L}+\Gamma_{R}}{2}\right)^{2}} d E
$$

This equation is derived analytically into Equation 15 (see main text) which, written into a constrained-range nonlinear curve-fitting program in LabVIEW, is fitted to all $\mathrm{i}-V_{\text {bias }}$ curves based on trust-region dogleg algorithm. The bias window under opposite polarities covers different ranges energetically and additional resonance levels may come in to contribute to currents under large biases, considering the small HOMO-LUMO gap in the well $\pi$-conjugated OPE3 moiety. To alleviate the complexities arising from large biases and focus only on the effect of $E_{\mathrm{F}, \text { substrate }}\left(V_{\mathrm{wk}}\right)$ on electrical properties, $\mathrm{i}-V_{\text {bias }}$ curves are fitted within $\pm 0.8 \mathrm{~V}$ instead of the full range. ${ }^{3}$ Figure 2 shows that the HOMO-LUMO gap of OPE3 bridged between gold electrodes is approximately $2 \mathrm{eV}$ (Figure 2), in a good agreement with the literature values. ${ }^{4}$ Hence, it is reasonable to carry out the fitting within the bias window of $\pm 0.8 \mathrm{~V}$.

Based on the fitting results for thiolated OPE3 by van der Zant and coworkers, ${ }^{5}$ the four parameters were assumed to be in the range of (i) $0 \mathrm{meV}<\Gamma_{\mathrm{L}(\mathrm{R})}<300 \mathrm{meV}$, (ii) $-1<\gamma<1$, and (iii) $-2 \mathrm{eV}<E_{\mathrm{FMO}}^{\mathrm{o}}$ $<2 \mathrm{eV}$ within which, accordingly, the optimized values of the fitting were constrained. For initial values within these ranges (including mixing combinations of the parameters, e.g., $10 \mathrm{meV}$ and $100 \mathrm{meV}$ for $\Gamma_{\mathrm{L}}(\mathrm{R})$, 0.0 and 0.5 for $\gamma$, and transition voltages derived from the fitting of Fowler-Nordheim plots $^{6}$ for $E_{\mathrm{FMO}}^{\mathrm{o}}$ ), the fitting resulted in those presented in Table S2.

Table S2 summarizes the fitting results. In the main text we state that the FMO shift under $V_{\mathrm{wk}}$ could not be ascribed to the variation of $\gamma$; the utmost energy shift, if considering the $i-V_{\mathrm{wk}}$ experiments, is calculated as follows: $V_{\text {bias }} \times($ maximum difference between $\gamma$, see Table S2 $) \times e=0.05\left(V_{\text {bias }}\right) \times(0.59-$ $0.44) \times e=7.5 \mathrm{meV} \sim 0.008 \mathrm{eV}$. Orbital energy $E_{\mathrm{FMO}}^{\mathrm{o}}\left(\mathrm{V} v s . E_{\mathrm{Ag} / \mathrm{AgCl}}\right)$ plotted in Figure $3 \mathrm{c}$ is derived by the simple arithmetic as follows: $V_{\mathrm{wk}}-\left(E_{\mathrm{FMO}}^{\mathrm{o}}-E_{\mathrm{F}, \text { substrate }}\right)$. The values of $|\delta \Delta|$ in Table S2 describe the change of the real part $\Delta$ of $\Sigma$ when modifying potentiostatted $V_{\mathrm{wk}}$ discretely, all yielded by taking the difference between $E_{\mathrm{FMO}}^{\mathrm{o}}\left(\mathrm{V} v s . E_{\mathrm{Ag} / \mathrm{AgCl}}\right)$ of two $V_{\mathrm{wk}}$ with a $0.25-\mathrm{V}$ increment. 
Table S2. Results of fitting $i-V_{\text {bias }}$ curves of OPE3 to the single-level Breit-Wigner formula.

\begin{tabular}{l||ccccccc}
\hline \multicolumn{7}{c}{$V_{\mathrm{wk}}\left(\right.$ Volt $\left.v s . E_{\mathrm{Ag} / \mathrm{AgCl}}\right)$} \\
\hline fitting parameters & -0.75 & -0.50 & $-0.25^{\mathrm{a}}$ & $0.00^{\mathrm{b}}$ & 0.25 & 0.50 & 0.75 \\
\hline$E_{\mathrm{FMO}}^{\mathrm{o}}-E_{\mathrm{F}, \text { substrate }}(\mathrm{eV})$ & 0.75 & 0.84 & $0.96 /-0.96$ & $1.02 /-1.02$ & -0.91 & -0.86 & -0.78 \\
coupling energy, $\Gamma_{L}(\mathrm{meV})$ & 10.8 & 11.2 & $12.8 / 12.9$ & $11.6 / 11.6$ & 11.9 & 13.0 & 13.2 \\
coupling energy, $\Gamma_{R}(\mathrm{meV})$ & 10.8 & 11.4 & $12.9 / 12.9$ & $11.6 / 11.6$ & 12.2 & 13.1 & 13.2 \\
FMO shift per volt, $\gamma$ & 0.51 & 0.50 & $0.55 / 0.44$ & $0.55 / 0.45$ & 0.54 & 0.56 & 0.59 \\
\hline orbital energy, $E_{\mathrm{FMO}}\left(\mathrm{V} v s . E_{\mathrm{Ag} / \mathrm{AgCl}}\right)$ & -1.51 & -1.34 & $-1.21 / 0.71$ & $-1.02 / 1.02$ & 1.16 & 1.36 & 1.53 \\
$|\delta \Delta|$ between neighboring $V_{\mathrm{wk}}$ & \multicolumn{2}{|c}{0.17} & 0.13 & $0.19 / 0.31$ & 0.14 & 0.20 & 0.17 \\
(shift in eV per 0.25-V change in $\left.V_{\mathrm{wk}}\right)$ & 0.17 &
\end{tabular}

a. Right to the slash are fitting parameters yielded by assuming HOMO as the dominant transporting FMO, and left to the slash are by assuming LUMO, instead. As described in the main text, the difference in the magnitude of energy offset $\left|E_{\mathrm{FMO}}^{\mathrm{o}}-E_{\mathrm{F} \text {,substrate }}\right|$ at $V_{\mathrm{wk}}$ of 0.00 and $-0.25 \mathrm{~V}$ is only $0.06 \mathrm{eV}$, which could be considered within the experimental error; therefore, the transporting FMO could be HOMO, but LUMO as the dominant FMO is also possible.

b. Right and left to the slash are fitting parameters yielded by assuming, respectively, HOMO and LUMO as the dominant transporting FMO. The magnitude of $E_{\mathrm{FMO}}^{\mathrm{o}}-E_{\mathrm{F}, \text { substrate }}$ at $V_{\mathrm{wk}}$ of $0.00 \mathrm{~V}$ is the largest, which implies that at some $V_{\mathrm{wk}}$ across $0.00 \mathrm{~V}$, there should be a transition of transporting FMO and ambiguity comes in when denoting the dominant one (i.e., LUMO or HOMO) at $V_{\mathrm{wk}}$ of $0.00 \mathrm{~V}$.

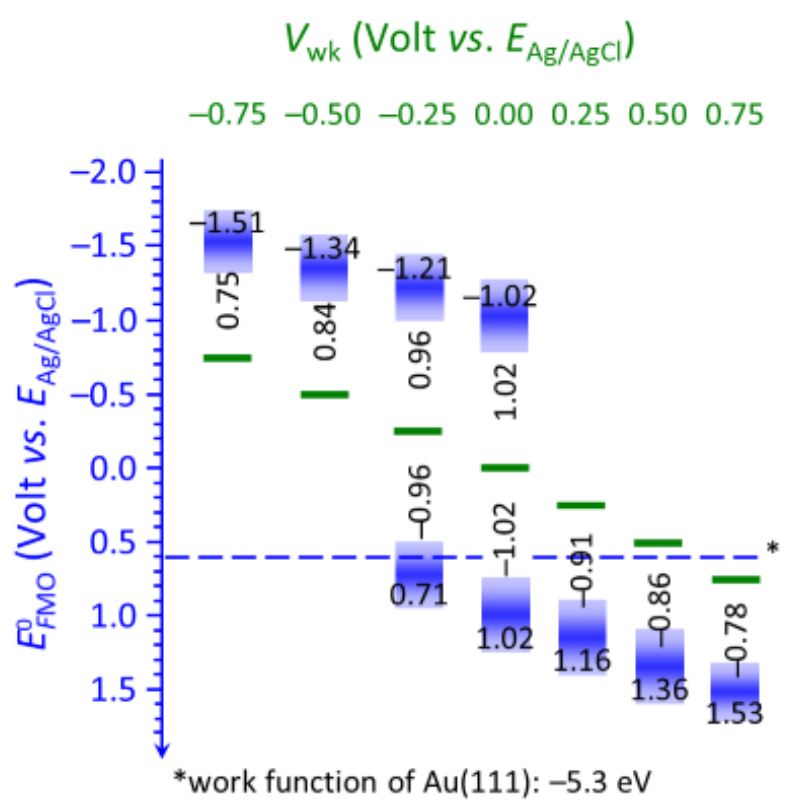

Figure S7. $V_{\mathrm{wk}}$-dependent $E_{\mathrm{FMO}}^{\mathrm{o}}\left(\mathrm{V} v s . E_{\mathrm{Ag} / \mathrm{AgCl}}\right)$ from the SLBW model. Fitting results of $E_{\mathrm{FMO}}^{\mathrm{o}}(\mathrm{V} v s$. $E_{\mathrm{Ag} / \mathrm{AgCl}}$, blue) from $i-V_{\mathrm{bias}}$ curves obtained at a series of fixed $V_{\mathrm{wk}}$ (green) from -0.75 to $+0.75 \mathrm{~V}$ versus $E_{\mathrm{Ag} / \mathrm{AgCl}}$. The blue-gradient boxes show the positions of $E_{\mathrm{FMO}}^{\mathrm{O}}$ (the central line) followed by standard deviations (the blue area) at every $V_{\mathrm{wk}} . E_{\mathrm{FMO}}^{\mathrm{o}}$ at $V_{\mathrm{wk}}$ of -0.25 and $0.00 \mathrm{~V}$ in this figure follow the propose in Table S2 (footnotes a and b) and are yielded via different initial setups in the Landauer fitting. $E_{\mathrm{FMO}}^{\mathrm{o}}$ at $V_{\mathrm{wk}}$ even more negative than the lower bound of the scan range in $i-V_{\mathrm{wk}}$ experiment (i.e., $V_{\mathrm{wk}}$ of -0.75 $\mathrm{V}$ ) is also fabricated based on the fitting results. The energy offsets are shown narrowing down at more negative $V_{\mathrm{wk}}$, implying some FMO (assumed to be LUMO) other than HOMO, is approaching $E_{\mathrm{F} \text {,substrate }}$. 
Table S3. Energy offset $\left(E_{\mathrm{FMO} @ V_{\mathrm{bias}}}-E_{\mathrm{F}, \text { substrate }}\right)$ derived via $E_{\mathrm{FMO}}^{\mathrm{o}}+\gamma e V_{\mathrm{bias}}-E_{\mathrm{F}}\left(E_{\mathrm{F}}\right.$ set to be zero $)$

\begin{tabular}{c|c|ccccccc}
\hline & \multirow{2}{*}{$V_{\text {bias }}$} & \multicolumn{7}{|c}{$V_{\mathrm{wk}}\left(\right.$ Volt $\left.v s . E_{\mathrm{Ag} / \mathrm{AgCl}}\right)$} \\
\cline { 3 - 9 } & & -0.75 & -0.50 & -0.25 & 0.00 & 0.25 & 0.50 & 0.75 \\
\hline \hline$E_{\mathrm{FMO} @ V_{\text {bias }}-E_{\mathrm{F}, \text { substrate }}}$ & $0.8 \mathrm{~V}$ & 0.34 & 0.44 & $0.52 /-1.31$ & $0.58 /-1.38$ & -1.34 & -1.31 & -1.25 \\
$\left(=E_{\mathrm{FMO}}^{\mathrm{o}}+\gamma e V_{\text {bias }}-E_{\mathrm{F}}\right)(e \mathrm{~V})$ & $-0.8 \mathrm{~V}$ & 1.16 & 1.24 & $1.40 /-0.61$ & $1.46 /-0.66$ & -0.48 & -0.41 & -0.31 \\
\hline
\end{tabular}

Notation:

$E_{\mathrm{FMO}}^{\mathrm{o}}$ : the energy level of FMO at zero bias indicated by the superscript.

$E_{\mathrm{FMO} @ V_{\text {bias }}}$ : The energy level of FMO under the applied bias.

$E_{\mathrm{F}, \text { substrate }}$ : The Fermi level of the substrate, viz., the working electrode.

$\gamma$ : FMO shift per volt of the applied bias. The values in Table $\mathrm{S} 2$ are utilized.

Table S4. Bias-induced shifts of $E_{\mathrm{FMO}}$ at $V_{\mathrm{bias}}$ of $0.8 \mathrm{~V}$ and $-0.8 \mathrm{~V}$.

\begin{tabular}{c|c||ccccccc}
\hline & \multirow{2}{*}{$V_{\text {bias }}$} & \multicolumn{7}{|c}{$V_{\mathrm{wk}}\left(\right.$ Volt $\left.v s . E_{\mathrm{Ag} / \mathrm{AgCl}}\right)$} \\
\cline { 3 - 9 } & & -0.75 & -0.50 & -0.25 & 0.00 & 0.25 & 0.50 & 0.75 \\
\hline \hline$E_{\mathrm{FMO}}^{\mathrm{o}}-E_{\mathrm{F}, \text { substrate }}{ }^{\mathrm{a}, \mathrm{b}}$ & $0.0 \mathrm{~V}$ & 0.75 & 0.84 & $0.96 /-0.96$ & $1.02 /-1.02$ & -0.91 & -0.86 & -0.78 \\
\hline$E_{\mathrm{FMO}}-\frac{1}{2}\left(E_{\mathrm{F}, \text { tip }}+E_{\mathrm{F}, \text { substrate }}\right)^{\mathrm{c}}$ & $0.8 \mathrm{~V}$ & 0.74 & 0.84 & $0.92 /-0.91$ & $0.98 /-0.98$ & -0.94 & -0.91 & -0.85 \\
& $-0.8 \mathrm{~V}$ & 0.76 & 0.84 & $1.00 /-1.01$ & $1.06 /-1.06$ & -0.88 & -0.81 & -0.71 \\
\hline$E_{\mathrm{FMO}}-\frac{1}{2}\left(E_{\mathrm{F}, \text { tip }}+E_{\mathrm{F}, \text { substrate }}\right)$ & $0.8 \mathrm{~V}$ & -0.01 & 0.00 & $-0.04 /+0.05$ & $-0.04 /+0.04$ & -0.03 & -0.05 & -0.07 \\
$-\left(E_{\mathrm{FMO}}^{\mathrm{O}}-E_{\mathrm{F}, \text { substrate }}\right)$ & $-0.8 \mathrm{~V}$ & 0.01 & 0.00 & $+0.04 /-0.05$ & $+0.04 /-0.04$ & 0.03 & 0.05 & 0.07 \\
\hline \multirow{2}{*}{$\begin{array}{c}\text { normalized bias-induced shifts of } E_{\mathrm{FMO}} \\
(\mathrm{eV} / \mathrm{V})\end{array}$} & $0.8 \mathrm{~V}$ & $-0.013^{\mathrm{d}}$ & 0.00 & $-0.050 /+0.063$ & $-0.050 /+0.050$ & -0.038 & -0.063 & $-0.088^{\mathrm{d}}$ \\
& $-0.8 \mathrm{~V}$ & -0.013 & 0.00 & $-0.050 /+0.063$ & $-0.050 /+0.050$ & -0.038 & -0.063 & -0.088 \\
\hline
\end{tabular}

a. $E_{\mathrm{FMO}}^{\mathrm{o}}$ is the orbital energy at zero bias. Note that, for the fitting of $E_{\mathrm{FMO}}^{\mathrm{o}}+\gamma e V_{\mathrm{bias}}-E_{\mathrm{F}}, E_{\mathrm{F}}$ (i.e., $E_{\mathrm{F}, \text { substrate }}$ or $\left.E_{\mathrm{wk}}\right)$ is set to be zero. This row is the results of the first row of Table 2.

b. This row shows the dependence of the orbital energy $E_{\mathrm{FMO}}^{\mathrm{o}}$ on the potentiostatted $E_{\mathrm{wk}}$ under zero bias.

c. $E_{\mathrm{F}}=\frac{1}{2}\left(E_{\mathrm{F}, \text { tip }}+E_{\mathrm{F} \text {,substrate }}\right)$, the definition of for two-terminal measurements (e.g., the dashed lines in Figure S8), is applied to facilitate a quick comparison with examples of bias-driven energy shifts reported in literature studies. Please be noted that there is fundamental difference between the schemes of applying the bias. Specifically, $E_{\mathrm{F}, \text { substrate }}$ is fixed for this present study while, for experiments of two-terminal electrodes, the potentials of both electrodes are scanning.

d. The negative (positive) sign of the shifts implies that the FMO offset to $E_{\mathrm{F}}$ (viz., $E_{\mathrm{FMO}}-$ $\left.\frac{1}{2}\left(E_{\mathrm{F}, \text { tip }}+E_{\mathrm{F} \text {,substrate }}\right)\right)$ decreases (increases) under positive bias compared to that under zero bias (where $E_{\mathrm{F}, \text { tip }}=E_{\mathrm{F} \text {,substrate }}$ such that the FMO offset to $E_{\mathrm{F}}$ could be written as $E_{\mathrm{FMO}}^{\mathrm{o}}-E_{\mathrm{F}, \text { substrate }}$ ); opposite modification of the offset occurs under negative bias. For example, in the case at $V_{\mathrm{wk}}$ of 0.75 $\mathrm{V}$ under the bias of $0.8 \mathrm{~V}$, the offset is modified by $(-0.088 \mathrm{eV} / \mathrm{V}) \times(0.8 \mathrm{~V})$; in other words, the offset decreases from $-0.78 \mathrm{eV}$ to $-0.85 \mathrm{eV}$; under the bias of $-0.8 \mathrm{~V}$, the offset is modified by $(-0.088 \mathrm{eV} / \mathrm{V})$ $\times(-0.8 \mathrm{~V})$, implying an increase of the offset (i.e., from $-0.78 \mathrm{eV}$ to $-0.71 \mathrm{eV})$. 


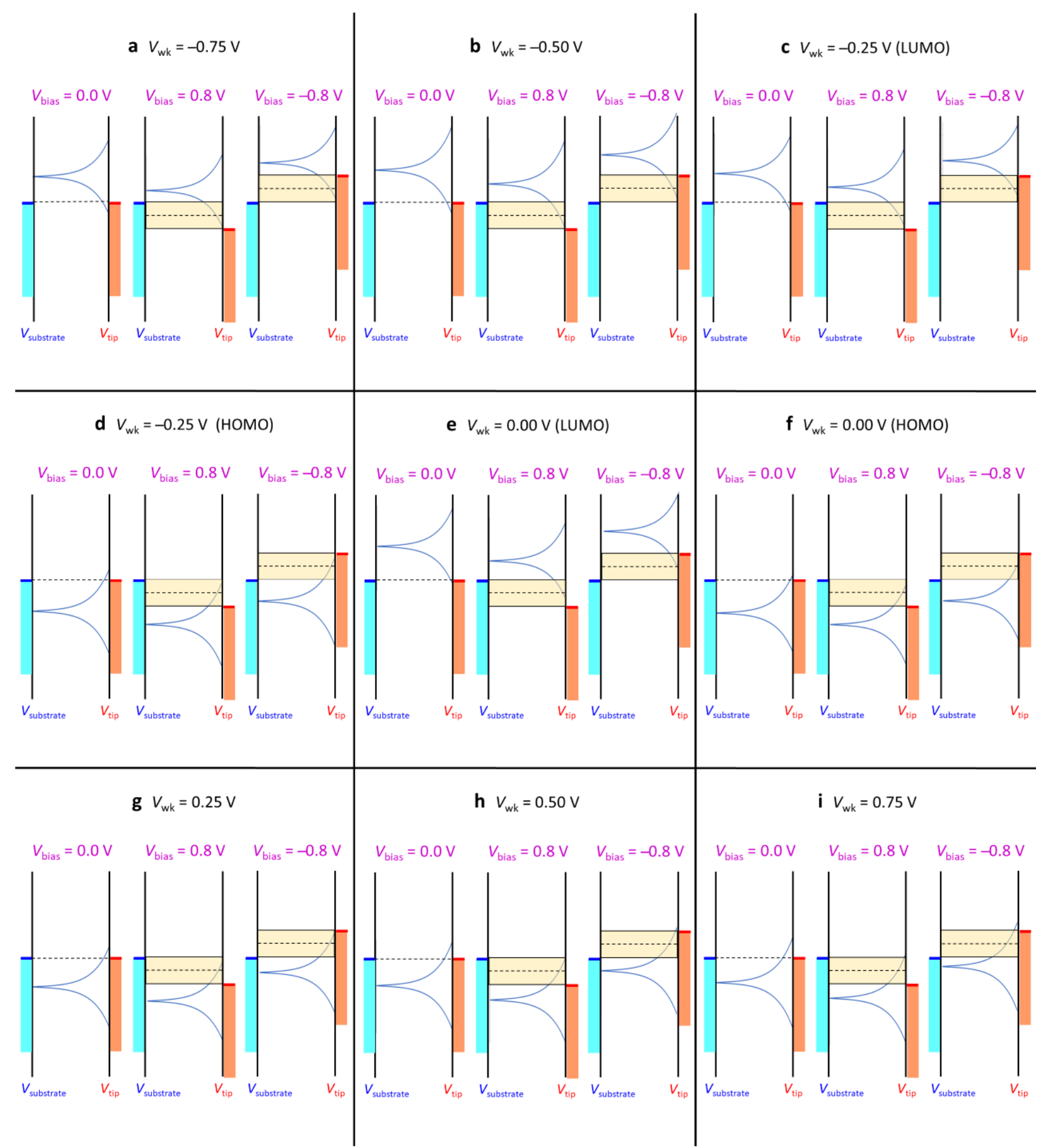

Figure S8. Transmission spectra for $i-V_{\text {bias }}$ reproduction of OPE3. To explain the rectification observed in the $i-V_{\text {bias }}$ plots (Figure S6), illustrative transmission spectra are drawn based on the fitting results (Table S2, energy offset, coupling constants, and the shift of the FMO per volt). The beige-shaded areas define the integration range into currents in the $i-V_{\text {bias }}$ curves. The areas and extents of asymmetry of transmission within the bias window are consistent with the $i-V_{\mathrm{bias}}$ experimental results, and this agreement validates the fitting. The dashed lines denote the level of $E_{\mathrm{F}}\left(=\frac{1}{2}\left(E_{\mathrm{F}, \text { tip }}+E_{\mathrm{F}, \text { substrate }}\right)\right)$ defined typically for two-terminal measurements (see Footnote c of Table S4). 


\section{DFT calculations.}

The calculations of electronic structures and transmission spectra were carried out using the DFTNEGF as implemented in the ATK package (QuantumATK 2017.2, Synopsys). ${ }^{7,8}$ A double- $\zeta$ polarization (DZP) basis set was used. An energy cutoff of 150 Ry defined the real space grid and Perdew-Zunger local density approximation (LDA) was chosen as the exchange-correlation functional. Electrodes were constructed from seven gold layers grown along the (111) direction, each containing $4 \times 4$ atoms. In the initial geometries, the $\mathrm{N}-\mathrm{Au}, \mathrm{S}-\mathrm{Au}$ and $\mathrm{C}-\mathrm{Au}$ distances were $2.26 \AA, 2.42 \AA$ and $2.00 \AA$ as used by previous works. $^{4,9}$ The geometries were obtained by relaxing atoms until forces were lower than 0.05 $\mathrm{eV} / \AA$. The geometries were optimized by relaxing whole molecules and contacted Au atoms except uncontacted $\mathrm{Au}$ atoms. The transmission spectra were calculated from each relaxed junction geometry.

a

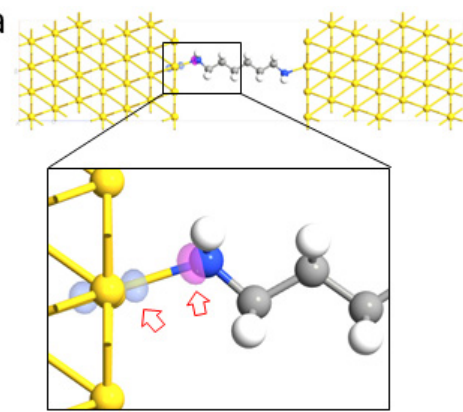

b

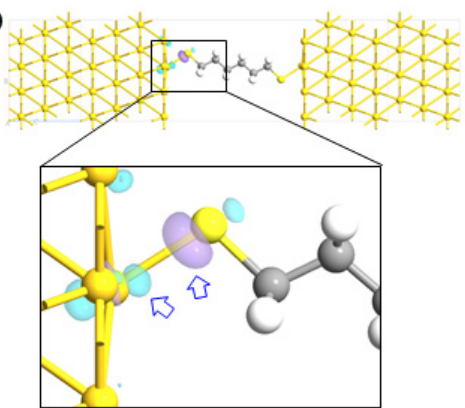

C

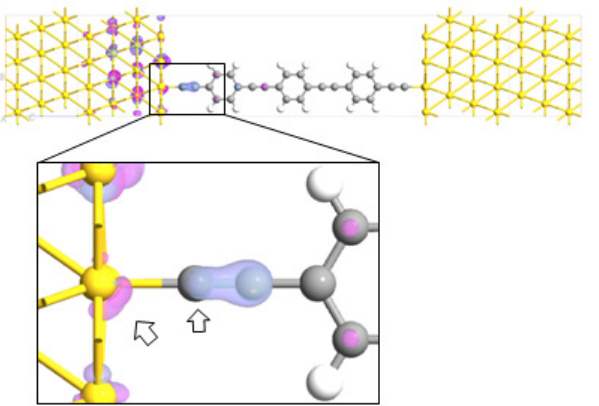

Figure S9. Optimized structures and isosurfaces of single-molecule junctions. Isosurfaces of electron charge density with the dominant eigenstates at zero bias $\left(E-E_{\text {Fermi }}=0\right)$ of the corresponding transmission spectra. (a) $\mathrm{H}_{2} \mathrm{~N}\left(\mathrm{CH}_{2}\right)_{6} \mathrm{NH}_{2}$. (b) $\mathrm{HS}\left(\mathrm{CH}_{2}\right)_{6} \mathrm{SH}$. (c) OPE3. The features indicated by the arrows suggest the development of headgroup-electrode hybridization. For clarity, the isovalues to present the results are 0.6 for $\alpha, \omega$-alkanes and 0.25 for OPE3.

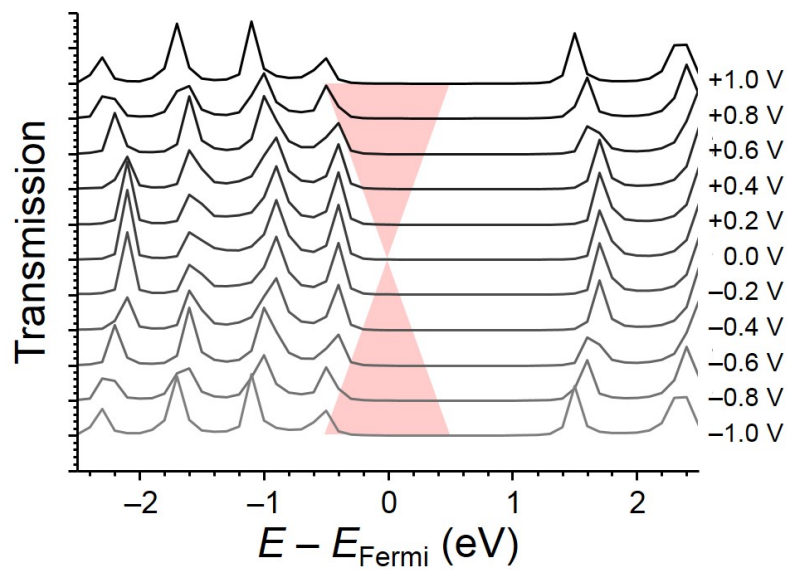

Figure S10. $V_{\text {bias }}$-dependent transmission spectra of OPE3. Linear-scale transmission spectra at $V_{\text {bias }}$ of $+1.0 \sim-1.0 \mathrm{~V}$ at $0.2 \mathrm{~V}$ intervals. The resolution of the spectra was $0.10 \mathrm{eV}$ due to the concerns of the computation load. The red triangles illustrate the windows of the applied bias voltage. Note that, upon the increase of $V_{\text {bias }}$, the peak position of HOMO shifted away from $E_{\text {Fermi }}$ as well. 


\section{Derivation of dependency of $G_{\text {contact }}$ and $\beta$ on the chemical potential of the electrode.}

The conductance of alkanediamines and OPE series decays exponentially with the number of repeated unit, which implicates that the super-exchange mechanism is feasible for both molecule series. The current, $I$, through a molecule junction can be expressed by Landauer formula:

$$
I=\frac{2 e}{h} \int_{-\infty}^{\infty} T(E)\left[f_{L}(E)-f_{R}(E)\right] d E
$$

where $f_{L(R)}(E)=\left[1+\left(E-\mu_{L(R)}\right) / \tau\right]^{-1}$ is the Fermi function of the left (right) electrode, $\mu_{L(R)}$ is the chemical potential of the left (right) electrode and $T(E)$ is the transmission function for a tunneling electron with energy $E$. Equation S1 can be properly simplified in concern of experiment conditions. In the study, the conductance of the molecules was measured at fixed and low bias $\left(V_{\text {bias }}=50 \mathrm{mV}\right)$. In addition of stepfunction-like property of the Fermi function, equation S1 can be deduced to calculate the molecular conductance as follows:

$$
G=\frac{2 e^{2}}{h} T\left(E_{F e r m i}\right)
$$

In the following argument, the dependency of $G_{\text {contact }}$ and $\beta$ with $E_{\text {Fermi }}$ is demonstrated by Simmons model and Newns-Anderson model via Equation S2.

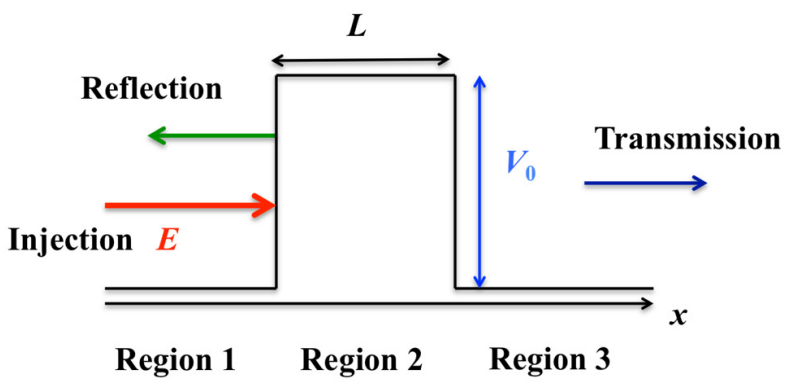

Figure S11. Schematic diagram of one-dimensional potential barrier. An electron with energy E strikes the rectangular barrier of height $V_{0}$ from the left and partially reflects and transmits.

\section{6-1. Simmons model}

The electrons are considered as penetrating through a potential barrier when passing through a molecule. Figure S11 is schematic diagram of a one-dimensional potential barrier. As an electron hits the barrier from the left, it partially reflects and transmits. It is noteworthy that it is sufficed to assume the potential profile is a perfect rectangle because the molecular conductance was measured at a fixed and small bias. By time-independent Schrödinger equation:

$$
\frac{-\hbar^{2}}{2 m} \frac{d^{2} \Psi}{d x^{2}}(x)+V(x) \Psi(x)=E \Psi(x)
$$

For region 1 and region $3, V(x) \equiv 0$; therefore, equation $\mathrm{S} 3$ can be rearranged as

$$
\frac{d^{2} \Psi}{d x^{2}}+\frac{2 m E}{\hbar^{2}} \Psi=0
$$

Equation S4 is a second order linear differential equation, and its solution are

$$
\Psi_{1}(x)=A_{1} \exp (i k x)+B_{1} \exp (-i k x)
$$




$$
\Psi_{3}(x)=A_{3} \exp (i k x)+B_{3} \exp (-i k x)
$$

for region 1 and 3 respectively, where $k=\sqrt{\frac{2 m E}{\hbar^{2}}}$. To study the reflection and transmission of an electron, one can assume $A_{1}=1, B_{1}=r$, and $A_{3}=t$; in addition, the electrons only propagate toward positive direction after transmitting through the barrier, so it is rational to let $\mathrm{B}_{3}=0$. For region $2, V(x) \equiv V_{0}$; equation S3 now reads

$$
\frac{d^{2} \Psi}{d x^{2}}+\frac{2 m\left(E-V_{0}\right)}{\hbar^{2}} \Psi=0
$$

Its solution is

$$
\Psi_{2}(x)=A_{2} \exp (i u x)+B_{2} \exp (-i u x)
$$

where $u=\sqrt{\frac{2 m\left(E-V_{0}\right)}{\hbar^{2}}}$. Here, $V_{0}$ is the height of the rectangular potential barrier and conceptually stands for the energy of the dominant transport molecular orbital. By the assumption stated above, it is noteworthy that $u$ is a pure imaginary number. By the fact that the total wavefunction must be continuous everywhere and so is its first derivative, the following equality should be satisfied

$$
\Psi_{1}(0)=\Psi_{2}(0) \& \frac{d \Psi_{1}}{d x}(0)=\frac{d \Psi_{2}}{d x}(0)
$$

and

$$
\Psi_{2}(L)=\Psi_{3}(L) \& \frac{d \Psi_{2}}{d x}(L)=\frac{d \Psi_{3}}{d x}(L)
$$

Then one can obtain

$$
1+r=A_{2}+B_{2} \& i k-i k r=i A_{2} u-i B_{2} u
$$

and

$$
\begin{gathered}
A_{2} \exp (i u L)+B_{2} \exp (-i u L)=t \exp (u k L) \\
i A_{2} u \exp (i u L)-i B_{2} u \exp (-i u L)=i t k \exp (i k L)
\end{gathered}
$$

By $\mathrm{S} 11$ and $\mathrm{S} 12, A_{2}$ and $B_{2}$ can be express in terms of $k$ and $u$

$$
A_{2}=\frac{1}{2}\left[\frac{k}{u}+1+\left(1-\frac{k}{u}\right) r\right] \& B_{2}=\frac{1}{2}\left[\frac{-k}{u}+1+\left(1+\frac{k}{u}\right) r\right]
$$

One can obtain a dual linear equation with unknowns, $r$ and $t$, by substitute S15 and S16 into S13 and $\mathrm{S} 14$, so the amplitude of the transmission wave, $t$, can be solved

$$
t=\frac{4 k u \exp (-i(k-u) L)}{(u+k)^{2}-(u-k)^{2} \exp (2 i u L)}
$$

Therefore, the transmission function can be calculated: 


$$
T(E)=|t|^{2}=t \cdot t^{*}=\frac{1}{\frac{V_{0}^{2}}{4 E\left(E-V_{0}\right)} \sinh ^{2}(i u L)+1}
$$

One assumes that the length of the barrier, $L$, is large enough such that $T(E)$ can be further approximated as

$$
T(E)=\frac{16 E\left(V_{0}-E\right)}{V_{0}^{2}} \exp (2 i u L)
$$

Substitute Equation S19 into Equation S2, one can conclude that

$$
G_{\text {contact }}=\frac{16 E_{\text {Fermi }}\left(V_{0}-E_{\text {Fermi }}\right)}{V_{0}^{2}} G_{0} \& \beta=\sqrt{\frac{2 m\left(V_{0}-E_{F e r m i}\right)}{\hbar^{2}}}
$$

where $G_{0}$ is the conductance quantum $\left(2 e^{2} / h\right)$. One can notice that the contact conductance is in second order with respect to Fermi level while tunneling decay constant is in square root relation. The results derived by Simmons model suggest that contact conductance is more sensitive to working potential than tunneling decay constant, consistent with experimental observations displayed in Figure $1 \mathrm{~b}$.

\section{6-2. Newns-Anderson model}

In the model, the transmission of the system is calculated by single-particle Green's function approach. The transmission function can be expressed as

$$
T(E)=\operatorname{Tr}\left(\Gamma_{L} G_{M}^{R} \Gamma_{R} G_{M}^{A}\right)
$$

where $G_{M}^{R(A)}=\left(E \cdot I-H_{M}-\left(\Sigma_{L}^{R(A)}-\Sigma_{R}^{R(A)}\right)\right)^{-1}$ is the retarded (advanced) molecular Green's function and $\Gamma_{L(R)}=i\left(\sum_{L(R)}^{R}-\Sigma_{L(R)}^{A}\right)$ is the left (right)-electrode coupling function. Here, $\Sigma_{L(R)}^{R(A)}$ stands for retarded (advanced) electrode self-energy from left (right) electrode and $H_{M}$ is the isolated molecular Hamiltonian.

Simplification of Equation S21 is available due to experimental setup. The molecular conductance was measured by EC-STM bj technique, so one can assume the self-energy of the electrodes only affects the anchoring group of molecules. Therefore, the electrode coupling function can be expressed as

$$
\Gamma_{L}=\left[\begin{array}{ccccc}
\Gamma_{L, l l} & 0 & \cdots & 0 & 0 \\
0 & 0 & \cdots & 0 & 0 \\
\vdots & \vdots & \ddots & \vdots & \vdots \\
0 & 0 & \cdots & 0 & 0 \\
0 & 0 & \cdots & 0 & 0
\end{array}\right] \& \Gamma_{R}=\left[\begin{array}{ccccc}
0 & 0 & \cdots & 0 & 0 \\
0 & 0 & \cdots & 0 & 0 \\
\vdots & \vdots & \ddots & \vdots & \vdots \\
0 & 0 & \cdots & 0 & 0 \\
0 & 0 & \cdots & 0 & \Gamma_{R, r r}
\end{array}\right]
$$

By the fact that retarded molecular Green's function is the conjugate transport of advanced molecular Green's function, S21 can be deduced as

$$
T(E)=\Gamma_{L, l l} \Gamma_{R, r r}\left|G_{M, l r}^{R}\right|^{2}
$$


To calculate the retarded molecular Green's function, tight-binding model is applied for Hamiltonian of both molecule series. For an alkanediamine with $n$ repeated methylene, its Hamiltonian can be expressed in the following form

$$
H_{M}=\left[\begin{array}{ccccccc}
\varepsilon_{a} & t_{m a} & 0 & \cdots & 0 & 0 & 0 \\
t_{m a} & \varepsilon_{m} & t_{m m} & \cdots & 0 & 0 & 0 \\
0 & t_{m m} & \varepsilon_{m} & \cdots & 0 & 0 & 0 \\
\vdots & \vdots & \vdots & \ddots & \vdots & \vdots & \vdots \\
0 & 0 & 0 & \cdots & \varepsilon_{m} & t_{m m} & 0 \\
0 & 0 & 0 & \cdots & t_{m m} & \varepsilon_{m} & t_{m a} \\
0 & 0 & 0 & \cdots & 0 & t_{m a} & \varepsilon_{a}
\end{array}\right]
$$

where $\varepsilon_{a}$ and $\varepsilon_{m}$ are on-site energy of the amine group and methylene group respectively, $t_{m a}$ is the hopping integral of amine group and methylene group, and $t_{m m}$ is the hopping integral of two adjacent methylene group. Note that $H_{M}$ is a $(n+2) \times(n+2)$ matrix. Therefore, the retarded molecular Green's function can be written as

$$
\begin{aligned}
G_{M}^{R} & =\left(E \cdot I-H_{M}-\Sigma^{R}\right)^{-1} \\
& =\left[\begin{array}{ccccccc}
E-\varepsilon_{a}-\Sigma_{L, l l}^{R} & -t_{m a} & 0 & \cdots & 0 & 0 & 0 \\
-t_{m a} & E-\varepsilon_{m} & -t_{m m} & \cdots & 0 & 0 & 0 \\
0 & -t_{m m} & E-\varepsilon_{m} & \cdots & 0 & 0 & 0 \\
\vdots & \vdots & \vdots & \ddots & \vdots & \vdots & \vdots \\
0 & 0 & 0 & \cdots & E-\varepsilon_{m} & -t_{m m} & 0 \\
0 & 0 & 0 & \cdots & -t_{m m} & E-\varepsilon_{m} & -t_{m a} \\
0 & 0 & 0 & \cdots & 0 & -t_{m a} & E-\varepsilon_{a}-\Sigma_{R, r r}^{R}
\end{array}\right]^{-1}
\end{aligned}
$$

One can calculate $G_{M, l r}^{R}$ by the following formula:

$$
A_{i j}^{-1}=\frac{(-1)^{i+j} C_{j i}}{\operatorname{det} A}
$$

where $C_{j i}$ is the cofactor of $A_{i j}$. In this case, $C_{r l}=(-1)^{n+1} t_{m a}^{2} t_{m m}^{n-1}$

One can assume $\left|E-\varepsilon_{a}-\Sigma_{L(R), l l(r r)}^{R}\right| \gg>\left|t_{m a(m m)}\right|$ and $\left|E-\varepsilon_{m}\right| \gg\left|t_{m m(m a)}\right|$, then $G_{M, l r}^{R}$ can be expressed as

$$
G_{M, l r}^{R}=\frac{(-1)^{n+1} t_{m a}^{2} t_{m m}{ }^{n-1}}{\left(E-\varepsilon_{a}-\Sigma^{R}{ }_{L, l l}\right)\left(E-\varepsilon_{a}-\Sigma^{R}{ }_{R, r r}\right)\left(E-\varepsilon_{m}\right)^{n}}
$$

By Equations S2 and S23, the conductance can be written as

$$
G=\frac{2 e^{2}}{h} \cdot \frac{\Gamma_{L, l l}\left|t_{m a}\right|}{\left|E_{F e r m i}-\varepsilon_{a}-\Sigma_{L}^{R}\right|^{2}} \cdot \frac{\Gamma_{R, r r}\left|t_{m a}\right|}{\left|E_{F e r m i}-\varepsilon_{a}-\Sigma_{R}^{R}\right|^{2}} \cdot\left|\frac{t_{m a}}{t_{m m}}\right|^{2} \cdot\left|\frac{t_{m m}}{E_{F e r m i}-\varepsilon_{m}}\right|^{2 n}
$$




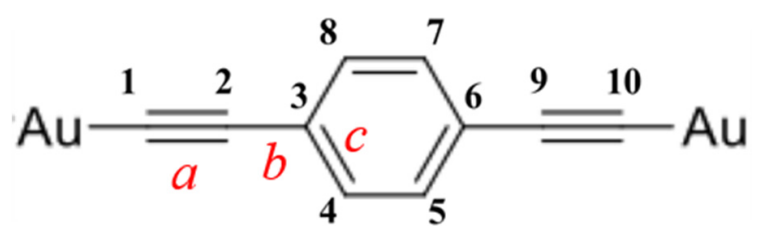

Similar methodology is applicable to OPE series. Symbols $a, b$ and $c$ are the hopping integrals between neighboring carbon atoms of triple bonds, single bonds, and those in the benzene ring. For OPEn, the molecular Hamiltonian is now written as

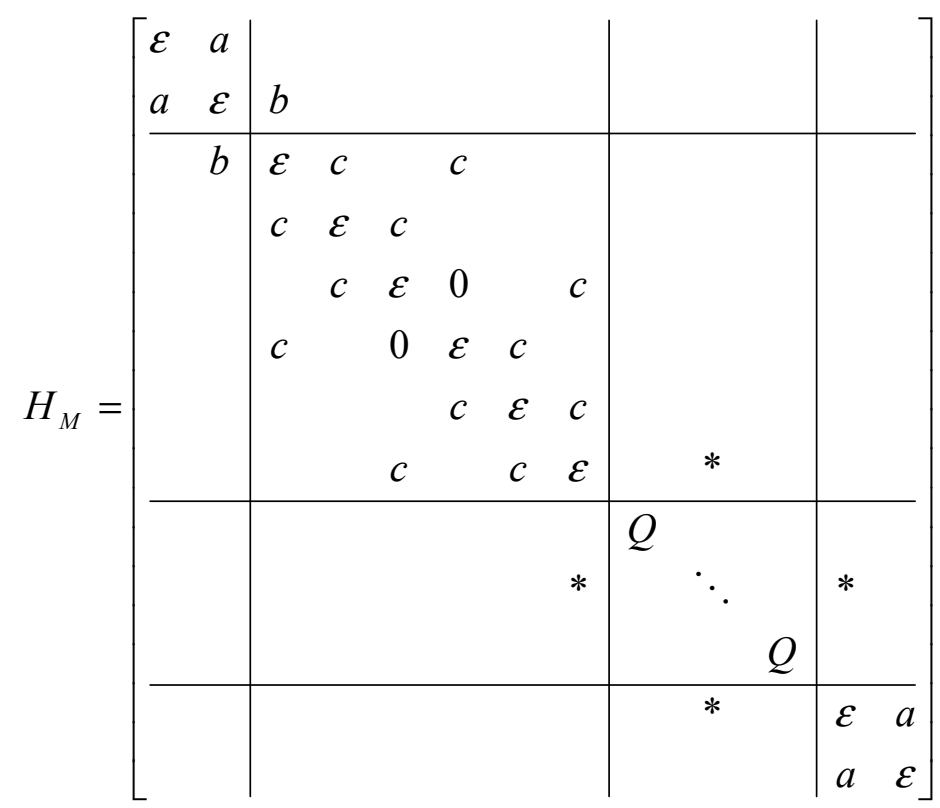

where $Q$ is the Hamiltonian of repeated unit. By the assumption that $\left|E-\mathcal{E}-\Sigma_{L(R), l(l r)}^{R}\right|$ is much greater than $a, b$ and $c$, one can calculate $G_{M, l r}^{R}$ and conductance in the same way as the case of alkanediamines. The result is also in the same mathematical form:

$$
G=\frac{2 e^{2}}{h} \frac{\Gamma_{L, l l} \Gamma_{R, r r} a^{2}}{\left|E_{\text {Fermi }}-\varepsilon-\Sigma_{L, l l}^{R}\right|^{2}\left|E_{\text {Fermi }}-\varepsilon-\Sigma_{R, r r}^{R}\right|^{2}}\left|\frac{u}{E_{F e r m i}-\varepsilon}\right|^{12 n}
$$

where $u^{6}=2 a b^{2} c^{3}$. It is therefore concluded that

$$
G_{\text {contact }}=\frac{\Gamma_{L, r r} \Gamma_{R, r r} a^{2}}{\left|E_{\text {Fermi }}-\varepsilon-\Sigma_{L, l l}^{R}\right|^{2}\left|E_{F e r m i}-\varepsilon-\Sigma_{R, r r}^{R}\right|^{2}} G_{0} \& \beta=12 \ln \left|\frac{E_{F e r m i}-\varepsilon}{u}\right|
$$

for OPE series. It is found that contact conductance is in fourth power with Fermi level while tunneling decay constant is in logarithm relation. Therefore, one can conclude that contact conductance changes more significantly with working potential than tunneling decay constant does. Note that $a, b$ and $c$ represent the hopping integrals of adjacent carbon atoms in the OPEn molecule; therefore, $u$ in equation S31 can be considered as average coupling strength between two adjacent carbons. Also note that the wide band approximation is an implicit assumption in the whole derivation because experimental condition was of low bias.

In summary, Simmons model and Newns-Anderson model are applied to derive the dependency of contact conductance and tunneling decay constant with the chemical potential of the electrodes. The 
results of both models indicate that the variation of the molecular conductance with working potential originates from the contact conductance more predominantly than tunneling decay constant. That is to say, the chemical potential of the electrode modulates the transport efficiency through metal-molecule contact more significantly than through molecular moiety.

\section{6-3. Glossary}

$T(E)$ : transmission function for a tunneling electron with energy $\mathrm{E}$

$f_{L(R)}(E)$ : Fermi function of the left (right) electrode

$\mu_{L(R)}$ : chemical potential of the left (right) electrode

$E_{\text {Fermi: }}$ Fermi level of the system

$G_{M}^{R(A)}:$ retarded (advanced) molecular Green's function

$G_{M, l r}^{R}$ : matrix element at row $l$ and column $r$ of $G_{M}^{R}$

$\Sigma_{L(R)}^{R(A)}$ : retarded (advanced) self-energy from the left (right) electrode

$V_{0}$ : height of the rectangular barrier

$\mathcal{E}_{a}:$ on-site energy of the amine group

$\varepsilon_{m}:$ on-site energy of the methylene group

$\varepsilon$ : on-site energy of the carbon atoms in OPE series

$t_{m a}$ : hopping integral between methylene group and amine group

$t_{m m}$ : hopping integral between two adjacent methylene group

$a, b$ and $c$ : hopping integral of adjacent carbon atoms in OPE series 


\section{References:}

(1) Ting, T.-C.; Hsu, L.-Y.; Huang, M.-J.; Horng, E.-C.; Lu, H.-C.; Hsu, C.-H.; Jiang, C.-H.; Jin, B.Y.; Peng, S.-M.; Chen, C.-h. Energy-Level Alignment for Single-Molecule Conductance of Extended Metal-Atom Chains. Angew. Chem. Int. Ed. 2015, 54, 15734-15738.

(2) Arroyo, C. R.; Leary, E.; Castellanos-Gómez, A.; Rubio-Bollinger, G.; González, M. T.; Agraït, N. Influence of Binding Groups on Molecular Junction Formation. J. Am. Chem. Soc. 2011, 133, 1431314319.

(3) Zotti, L. A.; Kirchner, T.; Cuevas, J. C.; Pauly, F.; Huhn, T.; Scheer, E.; Erbe, A. Revealing the Role of Anchoring Groups in the Electrical Conduction through Single-Molecule Junctions. Small 2010, $6,1529-1535$.

(4) Hong, W.; Manrique, D. Z.; Moreno-García, P.; Gulcur, M.; Mishchenko, A.; Lambert, C. J.; Bryce, M. R.; Wandlowski, T. Single Molecular Conductance of Tolanes: Experimental and Theoretical Study on the Junction Evolution Dependent on the Anchoring Group. J. Am. Chem. Soc. 2012, 134, 2292-2304.

(5) Frisenda, R.; Perrin, M. L.; Valkenier, H.; Hummelen, J. C.; van der Zant, H. S. J. Statistical Analysis of Single-Molecule Breaking Traces. Phys. Status Solidi B 2013, 250, 2431-2436.

(6) Beebe, J. M.; Kim, B.; Gadzuk, J. W.; Frisbie, C. D.; Kushmerick, J. G. Transition from Direct Tunneling to Field Emission in Metal-Molecule-Metal Junctions. Phys. Rev. Lett. 2006, 97, 026801.

(7) Smidstrup, S.; Stradi, D.; Wellendorff, J.; Khomyakov, P. A.; Vej-Hansen, U. G.; Lee, M.-E.; Ghosh, T.; Jónsson, E.; Jónsson, H.; Stokbro, K. First-Principles Green's-Function Method for Surface Calculations: A Pseudopotential Localized Basis Set Approach. Phys. Rev. B 2017, 96, 195309.

(8) Brandbyge, M.; Mozos, J.-L.; Ordejón, P.; Taylor, J.; Stokbro, K. Density-Functional Method for Nonequilibrium Electron Transport. Phys. Rev. B 2002, 65, 165401.

(9) Van Dyck, C.; Ratner, M. A. Molecular Rectifiers: A New Design Based on Asymmetric Anchoring Moieties. Nano Lett. 2015, 15, 1577-1584. 